\title{
Das Internet als Informations- quelle für den Aufbau von Berufswahlwissen: Wie Jugendliche es nützen, und wie nützlich sie die erhaltenen Informationen einschätzen
}

\section{Albert Düggeli, Katja Kinder und Wolfgang Kandzia}

Die vorliegende Untersuchung thematisiert den Zusammenhang zwischen in der Schule oder in der Freizeit aufgebauten Internetrecherchekompetenzen und der Einschätzung, nützliches Berufswahlwissen durch Internetrecherchen aufgebaut zu haben. Auf der Basis der Daten von Lernenden der achten Klassenstufe, die 2013 in der Schweiz im Rahmen der IEA ICIL-Studie (International Computer and Information Literacy) erhoben wurden $(N=1606)$ zeigt sich, dass eine zielorientierte Suche mit der Wabrnehmung einhergeht, nützliches Wissen aufgebaut zu haben. Die im schulischen Unterricht oder während des freizeitlichen Internetgebrauchs aufgebauten Recherchekompetenzen erwiesen sich, ebenso wie ein allfäliger Diskurs mit Lehrpersonen über das neu aufgebaute Berufswahlwissen, diesbezüglich als wenig bedeutsam. Die Befunde der Untersuchung werden theoretisch-konzeptionell im Rahmen ihrer wert-erwartungstheoretischen Bedeutung bzw. hinsichtlich ihrer Relevanz für die Berufswahl oder den Informatikunterricht insgesamt diskutiert.

\section{Einleitung und Fragestellung}

Der Übergang von der obligatorischen Schule in die postobligatorische Ausbildung bedeutet für Jugendliche, gemeinsam mit Eltern, Lehrpersonen oder Berufsberatenden eine möglichst passende Anschlusslösung zu finden. Dazu gehört, dass sie herausfinden, was sie inhaltlich gerne machen und welche Ausbildungswege ihnen angemessen sind. Auf dem Weg, eine berufsorientierte Ausbildungsentscheidung zu treffen, müssen Schulabgehende also Wissen zur eigenen Person und Wissen zur Berufs- und Arbeitswelt aufbauen (vgl. z. B. Düggeli \& Kinder, 2013; Hirschi, 2011; Ratschinski, 2008; Schweizerische Koordinationsstelle für Bildungsforschung [SKBF], 2018). Fehlt es, oder ist es lückenhaft, wird es für Jugendliche oft schwierig, verlässlich abschätzen zu können, ob sie beispielsweise die in einem Beruf zu leistenden Arbeiten richtig identifiziert haben, oder ob sie ausreichend über die für einen konkreten Beruf erforderlichen Kompetenzen verfügen. Hier unterstützt sie selbstbezügliches 
oder berufsbezogenes Wissen, weil es ihnen hilft, Berufswahlfragen besser strukturieren, Berufswahlziele differenzierter analysieren oder Handlungskonsequenzen klarer voraussehen zu können (Brown, Brooks \& Klostermann, 1994; Golisch, 2002; Hellberg, 2009). Dieses Berufswahlwissen ist im Übergangsprozess also von grosser Bedeutung und mit ihm sind es besonders auch die Informationsquellen, weil sie massgeblich darüber mitbestimmen, inwiefern Lernende substantielles Wissen aufbauen und damit ihren Berufswahlprozess zielführend voranbringen können. Eine mittlerweile weit verbreitete und auch curricular verankerte Informationsquelle ist der schulische Berufswahlunterricht (vgl. z. B. Deutschschweizer Erziehungsdirektorenkonferenz [D-EDK], 2016). Aber auch Gespräche mit Berufsberatenden, Lehrpersonen oder Eltern sind nach wie vor wichtige Informationsressourcen für Lernende, und nicht zuletzt stehen mittlerweile auch im Internet zahlreiche Informationen zu vielfältigen Aspekten der Berufswahl zur Verfügung. In diesem Medium suchen Jugendliche (12-19 Jahre) bis fast zu 10\% ihrer gesamten Nutzungszeit nach Informationen (Willemse, Waller, Süss, Genner \& Huber, 2012). Und mit spezifischem Blick auf die Berufswahl konnte Beinke (2008) in einer Untersuchung zeigen, dass etwa die Hälfte der Jugendlichen das Internet als Wissensquelle nutzt. Von den Sich-erfolgreich-Bewerbenden sind es etwa 56\%, von den sich Weniger-erfolgreich-Bewerbenden wird ein 15\% höherer Anteil berichtet, also etwa 71\%. Wenn Heranwachsende nun zum Aufbau ihres Berufswahlwissens das Internet als Informationsquelle nutzen, können sie besonders dann eine hohe Informationsqualität erreichen, wenn es ihnen gelingt, inhaltlich gehaltvolle Internetseiten von weniger substantiellen Angeboten zu unterscheiden, oder wenn sie die im Internet bereitgestellten Berufswahlinformationen inhaltlich klar selektieren, logisch strukturieren und produktiv in ihr bestehendes Wissen einbinden können (Düggeli, Kinder \& Bergamin, 2013). An dieser Stelle setzt der vorliegende Beitrag an. Er untersucht die Frage, wie nützlich Berufswahlsuchende im Internet recherchiertes Berufswahlwissen einschätzen, und zwar unter Einbezug von internetbezogenen Recherchekompetenzen, die sie in der Schule oder in der Freizeit aufgebaut haben. Vermittelnd werden das zielorientierte Vorgehen, die IT-bezogene Selbstwirksamkeit und eine mit den Eltern, Lehrpersonen oder Berufsberatenden allfällig vorgenommene Anschlusskommunikation über die recherchierten Informationen einbezogen.

\section{Theoretische Verortung}

Theoretisch-konzeptionell lässt sich diese Ausgangsfrage in zwei Richtungen einbetten. Zum einen mit Blick auf eine inhaltliche Ausdifferenzierung des Berufswahlwissens sowie dessen Verortung in gängigen berufs- und laufbahntheoretischen Konzepten. Zum anderen werden die Schule bzw. die Freizeit als zwei Handlungs- und Lernkontexte dargestellt, in denen Heranwachsende inter- 
netbezogene Recherchekompetenzen aufbauen können. Diese lassen sich mit dem zielorientierten Vorgehen und der Einschätzung, nützliche Informationen erhalten zu haben, in Verbindung bringen, wie in einem abschliessend dargestellten und die folgenden Analysen anleitenden Modell aufgezeigt wird.

\section{Berufswahlwissen}

Ins Berufswahlwissen fliessen nicht nur prozedurale Wissensaspekte ein, wie beispielsweise zu wissen, wie eine Bewerbung zu schreiben ist oder worauf bei einem Bewerbungsgespräch besonders geachtet werden muss, sondern es umfasst auch deklarative Wissensbestände, die aufzeigen, was Lernende besonders gut können bzw. in welchen Bereichen ihre Kompetenzen allenfalls weniger hoch ausgeprägt sind. Das ist deklaratives Selbstwissen. Es fokussiert als Teil des Berufswahlwissens die individuellen, berufsorientierten Kompetenzen und trägt somit dazu bei, dass Lernende sich selbst im Kontext ihrer Berufswahl differenziert wahrnehmen und folglich möglichst klar einschätzen können, inwiefern sie fähig sind, bestimmte Tätigkeiten qualitativ gehaltvoll auszuführen. Neben diesem Selbstwissen ist das Berufswissen der andere Teil des deklarativen Berufswahlwissens. Wenn Heranwachsende also gerne feststellen möchten, in welchen Berufsfeldern sie ihre Kompetenzen einsetzen, oder durch welche Berufstätigkeiten sie bestehende Kompetenzen weiter entwickeln können, benötigen sie Berufswissen. Es beschreibt neben konkreten manuellen Tätigkeiten, die vielleicht in einem Beruf bedeutsam sind, gegebenenfalls auch soziale Aspekte, auf denen das berufliche Tätig-Sein in einer spezifischen Branche hauptsächlich beruht. Berufswissen widerspiegelt also in gewisser Weise kontextuelle Aspekte der Berufswahl, während das Selbstwissen eher die personale Ebene fokussiert. Beide Bereiche lassen sich aber nicht nur als zentrale Perspektiven des Berufswahlwissens verstehen, sondern sind auch in unterschiedlichen theoretischen Konzeptionen und Modellen zur beruflichen Entwicklung bedeutsam.

\section{Das Berufswahlwissen in ausgewählten Berufswahlkon- zepten}

Die Unterscheidung von Berufs- und Selbstwissen und damit die Differenzierung in eher personale bzw. umweltbezogene Aspekte der Berufswahl, finden sich besonders prominent in passungstheoretischen Berufs- und Laufbahnkonzepten. Diese gehen davon aus, dass ein hoher Passungsgrad zwischen individuellen Voraussetzungen (Person) und berufsfeldspezifischen Anforderungen (Umwelt) die Grundlage für eine gelingende Berufswahl darstellt (vgl. z. B. Holland, 1997). Aber auch in lerntheoretischen Modellen wird der Aufbau von diesen beiden Wissensfacetten relativ prominent vertreten, weil die Berufswahl hier als konkrete Lerngelegenheit verstanden wird, die es ermöglicht, zu diesen beiden Bereichen spezifisches Wissen aufbauen und in der Folge situative Handlungsanforderungen erfolgreich bewältigen zu können (Mitchell \& Krumboltz, 1994). Diese beiden Wissensbereiche sind aber auch in stärker 
berufsbiografisch ausgerichteten Modellen bedeutsam (Lent, Brown \& Hackett, 2002) bzw. finden sich in entscheidungstheoretischen Konzepten an zentraler Stelle wieder. Gerade in letzteren ist differenziertes Wissen deshalb grundlegend, weil der Übergang von der obligatorischen in die nachobligatorische Ausbildung in gewisser Weise strukturell festgelegt und damit der Zeitpunkt der Entscheidung systemisch gegeben ist. Die Lernenden haben, wenn man es so betrachtet, nicht unbegrenzt Zeit, die Transition zu bewerkstelligen, sondern finden idealerweise nach der obligatorischen Schulzeit eine direkte Anschlusslösung auf der Sekundarstufe II. Insofern erweist sich ein möglichst zielgerichtetes Vorgehen für einen gelingenden Transitionsprozess als vorteilhaft. Dies heisst, mit Blick auf das Berufswahlwissen ganz allgemein, dass erstens beides, berufsbezogenes oder selbstbezogenes Wissen in mehreren berufswahltheoretischen Zugängen relevant ist, und dass Jugendliche es sich auf verschiedenen Wegen erschliessen können, beispielsweise indem sie ihre Eltern fragen oder sich im Berufsbildungszentrum informieren, oder auch, wenn sie sich im Internet nach berufswahlrelevanten Informationen umsehen.

\section{Internetrecherchen und Wissensaufbau}

Internetrecherchen führen Heranwachsende bekanntlich nicht nur im Kontext schulischen Lernens durch, sondern sie nutzen das Internet genauso als Informationsquelle während ihren freizeitlichen Aktivitäten (vgl. z. B. Fraillon, Ainley, Schulz, Friedman \& Gebhardt, 2014; Konsortium PISA.ch, 2014). Schulische und freizeitliche Nutzungsformen sind also Lerngelegenheiten, um Recherchekompetenzen aufzubauen, so dass es Heranwachsenden möglichst gut gelingt, inhaltlich substantielle Internetseiten auffinden und zielbezogen durch diese navigieren zu können. Recherchekompetenzen unterstützen die Lernenden also darin, nicht nur rein zufällig an vielleicht eher vagen Informationen vorbei zu kommen, sondern sie helfen ihnen, systematisch jenes Sachwissen zu erkunden, das für die Klärung ihrer Sachfragen grundlegend ist. Dabei ist tendenziell davon auszugehen, dass der Aufbau von Recherchewissen im schulischen Umfeld eher in formalisierten Lernsettings stattfindet, beispielsweise, wenn Lernende während des Unterrichts zu einem Thema nach Wissen recherchieren müssen, oder wenn sie aufgefordert werden, bestimmte Computer-Manipulationen bei der Nutzung von Suchmaschinen anzuwenden. Ebenfalls geht es im schulischen Kontext oft darum zu lernen, wie recherchierte Informationen inhaltlich angemessen und vor allem zieladäquat wiedergegeben werden können (D-EDK, 2016). Recherchekompetenzen im ausserschulischen Umfeld hingegen scheinen sich eher durch eine Art bedarfsorientierte Nutzung in so genannten ad-hoc-Such-Situationen zu entwickeln. Dies ist beispielsweise der Fall, wenn Heranwachsende in einem ihnen wenig vertrauten Ort Ausgehlokale finden wollen, oder wenn sie wissen möchten, wie weit von ihnen entfernt der nächstgelegene Bahnhof zu finden ist. In beiden Recherchekontexten, im schulischen und im freizeitlichen, ist es aber möglich, dass Jugendliche mit Informa- 
tionen konfrontiert werden, die nicht direkt mit ihrem Wissensziel vereinbar sind und deshalb nur wenig dazu beitragen, gesuchtes Wissen aufbauen oder zu klärende Fragen beantworten zu können. Gerade weil das im Internet zugängliche Wissen auf mehreren Wegen erschlossen werden kann und meist auch mehrere weitere Suchoptionen eröffnet, ist es wichtig, das eigene Vorgehen mit Blick auf die Zielfokussierung oder hinsichtlich allenfalls angezeigten Suchoptimierungen zu überprüfen. Erfolgreiche Internetrecherchen erfordern also, fokussiert zu bleiben, damit ein möglichst zielorientierter Wissensaufbau erfolgen kann. Genauso erfordern aber erfolgreiche Internetrecherchen eine hohe IT-Selbstwirksamkeit, und damit die grundlegende Überzeugung, fähig zu sein, das Internet so nutzen zu können, dass die angestrebten Ziele auch erreicht werden. Die IT-Selbstwirksamkeit ist also etwas weniger konkret an das Berufswahlwissen oder an andere Inhaltsbereiche gebunden, zu denen Wissen recherchiert wird, ist aber trotzdem als ein zentrales Merkmal zu betrachten, das die Lernenden beim Aufbau von möglichst nützlichem Berufswahlwissen unterstützt. Und Unterstützung erfahren die Lernenden nicht zuletzt auch durch die Möglichkeit, recherchierte Informationen mit ihnen wichtigen und thematisch kompetent erscheinenden Personen austauschen können. Lehrpersonen beispielsweise oder Eltern sind für die Jugendlichen meist wichtige Bezugspersonen, mit denen sie ihre Berufswahl erörtern, bzw. die ihnen oftmals beratend und unterstützend beim Aufbau von Berufswahlwissen zur Seite stehen. Dieser Austausch lässt sich als eine Art Anschlusskommunikation verstehen, die nach erfolgten Recherchetätigkeiten möglich wird, so dass sich, wie das nachfolgende Modell zusammenfassend zeigt, dieses Merkmal nach den Recherchetätigkeiten verorten lässt, vermittelnd zwischen der zielorientierten Nutzung und der eingeschätzten Nützlichkeit der Suchergebnisse (vgl. Abb. 1).

\section{Internetrecherchen zum Aufbau von Berufswahlwissen}

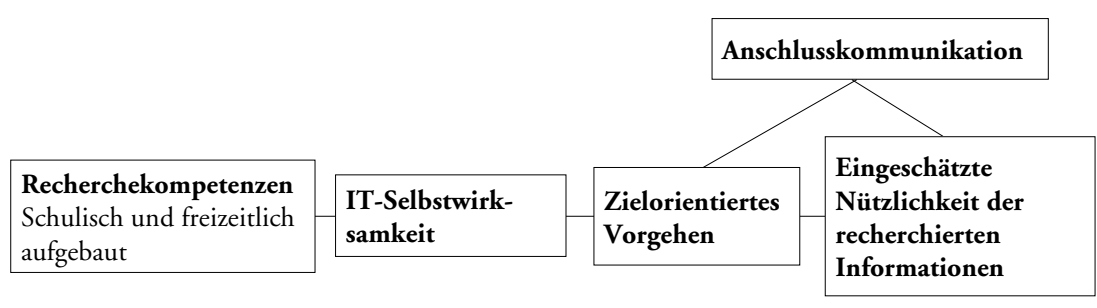

Abb. 1: Ausgangsmodell zum Zusammenhang von Internet-Recherchen und dem Aufbau von Berufswahlwissen. 


\section{Die Untersuchung}

Die Fragestellung, inwiefern in der Schule oder in der Freizeit erworbene Recherchekompetenzen mit der eingeschätzten Nützlichkeit von recherchiertem Berufswahlwissen zusammenhängen, und inwiefern diese Kompetenzen über die IT-Selbstwirksamkeit bzw. eine zielorientierte Nutzung des Internets vermittelt werden, basiert auf den folgenden Hypothesen. Die schulisch und die freizeitlich aufgebauten Recherchekompetenzen hängen direkt (1) und indirekt positiv mit der eingeschätzten Nützlichkeit der recherchierten Informationen zusammen (2); im indirekten Modell wird die zielorientierte Nutzung als Mediator einbezogen. Direkte positive Zusammenhänge bestehen ebenfalls zwischen den in beiden Bereichen aufgebauten Recherchekompetenzen und der zielorientierten Nutzung (3). Diese werden über die Selbstwirksamkeit vermittelt (4). Die Anschlusskommunikation mediiert die positive Assoziation zwischen der zielorientierten Nutzung und der eingeschätzten Nützlichkeit (5).

\section{Methode}

Zur Klärung dieser Hypothesen wurden Daten analysiert, die im Rahmen der IEA-ICIL-Studie in der Schweiz entweder im internationalen Untersuchungsteil oder im nationalen Zusatzmodul im ersten Halbjahr 2013 erhoben wurden (CIL; Computer and Information Literacy) (Fraillon, Ainley, Schulz, Friedman \& Gebhardt, 2014). Im nationalen Zusatzmodul beantworteten Achtklässler und Achtklässlerinnen während der Schulzeit am Computer Fragen zu ihren internetbezogenen Aktivitäten im Rahmen ihrer Berufswahl $(\mathrm{N}=3225)$. Sie wurden beispielsweise gefragt, ob sie beim Aufbau von Selbst- bzw. Berufswissen zielorientiert vorgehen (Zielorientierung), oder wie sie die Qualität der erhaltenen Informationen einschätzen (Nützlichkeit). Auch wurde erhoben, ob sie die erhaltenen Suchergebnisse mit ihnen nahestehenden Personen besprechen (Anschlusskommunikation). Aus dem internationalen Teil der Studie wurden Angaben zu den in der Schule und in der Freizeit aufgebauten Recherchekompetenzen entnommen. Ebenfalls stammt die Skala zur IT-bezogenen Selbstwirksamkeit aus diesem Untersuchungsteil.

\section{Stichprobe}

In die Analysen der vorliegenden Untersuchung flossen die Angaben von mindestens 1310 und höchstens 1606 Lernenden ein, die keinen gymnasialen Schultyp besuchen. Im Unterschied zu den Lernenden, die bereits ins Gymnasium übergetreten sind, deren postobligatorische Ausbildung also in gewisser Weise vorgespurt ist, stehen die anderen gegen Ende der obligatorischen Schule vor einem Übertritt und können deshalb von einem allfälligen Berufswahlwissen besonders profitieren. Für die Analyse des Strukturgleichungsmodells wurden nur Daten verwendet, wenn mindestens $50 \%$ der Indikatorvariablen jedes latenten Konstrukts vorhanden waren. Dies führte zum Ausschluss von 44 
Personen. Weitere 6 Personen wurden anhand der quadrierten Mahalanobisdistanz als multivariate Ausreisser identifiziert und ebenfalls aus der Stichprobe ausgeschlossen. Somit verblieben für die Berechnung des Strukturgleichungsmodells 1556 Lernende im Sample. Von den gesamthaft in dieser Studie untersuchten 1606 Lernenden waren 52.4\% Männer und 47.6\% Frauen. $12.8 \%$ der befragten Heranwachsenden gab an, bezüglich Berufswahl noch keine klare Idee zu haben, also noch diffus suchend zu sein. Bei $43.7 \%$ hat sich die Ausrichtung auf eine Anschlusslösung bereits konkretisiert und 22.6\% gaben zum Zeitpunkt der Erhebung an, einen Entscheid getroffen zu haben und einen Ausbildungsplatz zu suchen. $17.6 \%$ hatten bereits eine Anschlusslösung, befanden sich also in einer Art konsolidierenden Phase der Berufswahl (Herzog, Neuenschwander \& Wannack, 2006).

\section{Messinstrumente}

Die verwendeten Skalen wurden im Rahmen der IEA ICIL-Studie entweder für den internationalen oder nationalen Teil der Studie entwickelt und geprüft (Fraillon, Schulz, Friedman, Ainley \& Gebhardt, 2015). Sie lassen sich wie folgt operationalisieren:

IT-Lernen in der Schule: Das in der Schule vermittelte IT-Anwendungswissen wurde durch zwei Fragen erfasst, die ermittelten, wie häufig das Internet zur Informationssuche für die Schule genutzt wird, und wie häufig die Lernenden auf Online-Enzyklopädien zugreifen $(\alpha=.80)$. Die fünf möglichen Antwortformate waren: «Nie»; «Weniger als einmal im Monat»; «Mindestens einmal im Monat aber nicht jede Woche»; «Mindestens einmal die Woche aber nicht jeden Tag»; «Jeden Tag».

IT-Nutzung in der Freizeit: Diese Skala erfasst die IT-Anwendung zum Zwecke der Informationsrecherche und Informationsdarbietung ausserhalb der Schule $(\alpha=.77)$. Mit acht Items wurde ermittelt, wie oft Jugendliche das Internet ausserhalb der Schule dafür verwenden. Die fünf möglichen Antwortformate waren: «Nie»; «Weniger als einmal im Monat»; «Mindestens einmal im Monat aber nicht jede Woche»; «Mindestens einmal die Woche aber nicht jeden Tag»; «Jeden Tag».

IT-Selbstwirksamkeit: Diese Skala erfasst die wahrgenommene Selbstwirksamkeit mit Blick auf die Bearbeitung von sieben komplexeren Aufgaben im Kontext der Computeranwendung $(\alpha=.81)$. Gefragt wird beispielsweise, ob die Lernenden es sich zutrauen, eigenständig Datenbanken anzulegen oder die Einstellungen ihres Computers zu ändern, um ihn leistungsfähiger rechnen zu lassen. Die Antwortformate waren: «Ich kann das ausführen»; «Ich kann herausfinden, wie man das macht»; «Ich denke nicht, dass ich das kann».

Zielorientierte Nutzung: Mittels dieser Skala wurde erfasst, inwiefern die Suche nach Informationen zum Berufs- oder Selbstwissen zielorientiert erfolgt $(\alpha=.63)$. Ein Beispielitem lautet: «Um herauszufinden, was es braucht, um einen 
bestimmten Ausbildungsweg zu verwirklichen, habe ich im Internet gezielt nach den zu erfüllenden Zugangsvoraussetzungen gesucht». Als Antwortkategorien standen vier Abstufungen zur Verfügung: «Trifft nicht zu»; "Trifft eher nicht zu»; «Trifft eher zu» und «Trifft zu».

Anschlusskommunikation: Die Anschlusskommunikation erfasste die Angabe, ob Lernende eine im Internet durchgeführte Informationssuche mit Eltern, Lehrpersonen oder Berufsberatenden besprochen haben $(\alpha=.89)$. Mögliche Antwortformate waren: «Habe ich gemacht»; "Habe ich nicht gemacht».

Eingeschätzte Nützlichkeit: Diese Skala erhebt, ob die durchgeführten Internet-Recherchen zum Aufbau von Selbst- und Berufswissen als nützlich empfunden wurden $(\alpha=.71)$. Die Antworten wurden auf vier vierstufigen Likert-Skalen abgebildet, mit den Abstufungen «Trifft nicht zu»; «Trifft eher nicht zu»; «Trifft eher zu» und «Trifft zu».

Kontrollvariablen: Das Alter der Versuchspersonen, ihr sozioökonomischer Hintergrund (Ganzeboom, De Graaf, Treiman, \& De Leeuw, 1992) sowie ihr Geschlecht wurden erhoben, um den Einfluss dieser Merkmale auf die Ergebnisse zu kontrollieren.

\section{Resultate}

Die Ergebnisse zeigen zunächst bivariate Zusammenhänge zwischen den untersuchten Merkmalen. Anschliessend machen Gruppenvergleiche sichtbar, inwiefern sich Lernende mit eher tiefen, mittleren oder hohen Nützlichkeitseinschätzungen auf den erfassten Merkmalen unterscheiden. Abschliessend erfolgt die Analyse eines multivariaten Zusammenhangsmusters zwischen den untersuchten Merkmalen auf der Basis eines Strukturmodells.

\section{Bivariate Merkmalszusammenhänge}

Die Analyse der bivariaten Merkmalszusammenhänge erfolgte mit SPSS 25. Die positive Verbindung zwischen der eingeschätzten Nützlichkeit der recherchierten Informationen und der zielorientierten Nutzung ist mittelstark und verglichen mit allen anderen Korrelationen am grössten (vgl. Tab. 1).

Ebenfalls stehen die in der Schule erlernten oder während der Freizeit angeeigneten Recherchekompetenzen, sowie die IT-Selbstwirksamkeit mit der eingeschätzten Nützlichkeit in statistisch bedeutsamen Zusammenhängen. Diese sind aber mit einem Koeffizienten von $\mathrm{r}<.2$ nur schwach und werden deshalb hier nicht vertiefend diskutiert. Besonders hervorzuheben sind hingegen die Zusammenhänge zwischen der IT-Selbstwirksamkeit und den anderen Variablen. Sie hängt positiv und mittelstark mit der freizeitlichen Nutzung des Internets als Wissensquelle zusammen. Ebenfalls positiv aber nur schwach ist ihr Zusammenhang mit der zielorientierten Nutzung sowie schwach-negativ mit dem Geschlecht. 
Tab. 1: Bivariate Zusammenhänge der untersuchten Merkmale (Korrelationskoeffizient nach Pearson).

\begin{tabular}{|c|c|c|c|c|c|c|c|c|c|c|}
\hline Merkmale & $\mathrm{N}$ & M & SD & (1) & (2) & (3) & $(4)$ & (5) & (6) & (7) \\
\hline (1) Geschlecht $(\mathrm{m}=0)$ & 1606 & .48 & .50 & & & & & & & \\
\hline (2) HISEI & 1590 & 52.0 & 16.56 & -.03 & & & & & & \\
\hline (3) IT-Schule & 1568 & 45.7 & 10.3 & .00 & -.02 & & & & & \\
\hline (4) IT-Freizeit & 1579 & 45.7 & 8.88 & $-.09^{*}$ & -.04 & .05 & & & & \\
\hline (5) IT-Selbstwirksamkeit & 1570 & 47.5 & 9.9 & $-.26^{* *}$ & -.02 & $.07^{* *}$ & $.33^{* *}$ & & & \\
\hline (6) Zielorientierte Nutzung & 1311 & 2.8 & .60 & $-.09^{*}$ & $-.09^{*}$ & $.07^{* *}$ & $.17^{* *}$ & $.24^{* *}$ & & \\
\hline (7) Anschlusskommunikation & 1310 & .29 & .24 & $.13^{* *}$ & -.01 & $.06^{*}$ & $-.08^{* *}$ & $-.13^{* *}$ & .03 & \\
\hline (8) Nützlichkeit & 1311 & 2.6 & .67 & $-.06^{*}$ & -.04 & $.12^{* *}$ & $.09^{* *}$ & $.12^{* *}$ & $.44^{* *}$ & .05 \\
\hline
\end{tabular}

Eine höhere IT-Selbstwirksamkeit ist also eher mit dem männlichen Geschlecht assoziiert. Überdies zeigen sich keine namhaften Verbindungen der untersuchten Variablen zur sozialen Herkunft (HISEI). Wichtig ist vielleicht noch der Hinweis darauf, dass die Anschlusskommunikation mit der IT-Selbstwirksamkeit negativ zusammenhängt. Je höher also die Anschlusskommunikation, desto weniger hoch scheint die IT-Selbstwirksamkeit ausgeprägt.

\section{Gruppenvergleiche}

Der zweite, deskriptive Analyseschritt stellt die wahrgenommene Nützlichkeit ins Zentrum. Gebildet wurden drei Gruppen; eine erste mit vergleichsweise niedrigen Ausprägungen der eingeschätzten Nützlichkeit $(319<=n<=419)$ $(\mathrm{M}=1.84 ; \mathrm{SD}=.39)$, eine zweite mit vergleichsweise mittleren $(347<=\mathrm{n}<=405)$ $(\mathrm{M}=2.60 ; \mathrm{SD}=.15)$, und eine dritte mit eher hohen Ausprägungen auf diesem Merkmal $(460<=n<=514) \quad(M=3.30 ; S D=.33)$. Verglichen wurden die drei Gruppen bezüglich des in der Schule erworbenen IT-Recherchewissens, der IT-Nutzung in der Freizeit, der IT-Selbstwirksamkeit, der zielorientierten Nutzung sowie der Anschlusskommunikation. Die mittels einer ANOVA berechneten Gruppenvergleiche (Bonferroni-Post-hoc-Kontraste) sind mit Ausnahme der Anschlusskommunikation signifikant (vgl. Tab. 2).

Mit Blick auf das IT-Lernen in der Schule heisst dies, dass die Gruppe mit der vergleichsweise geringsten Nützlichkeitseinschätzung bedeutsam weniger häufig in der Schule IT-Recherchekompetenzen aufbaut. Mit Blick auf die freizeitliche Nutzung ist es etwas anders. Hier weist die Gruppe, die am wenigsten Nützlichkeit berichtet, den höchsten Nutzungswert aus. Und auch hinsichtlich der Selbstwirksamkeit weist die Gruppe mit der geringsten Nützlichkeitseinschätzung den höchsten Wert aus. 
Tab. 2: Gruppenvergleiche zwischen der eingeschätzten Nützlichkeit (TerzilGruppen) und den anderen untersuchten Merkmalen (ANOVA; post-hoc Gruppenvergleiche Bonferroni).

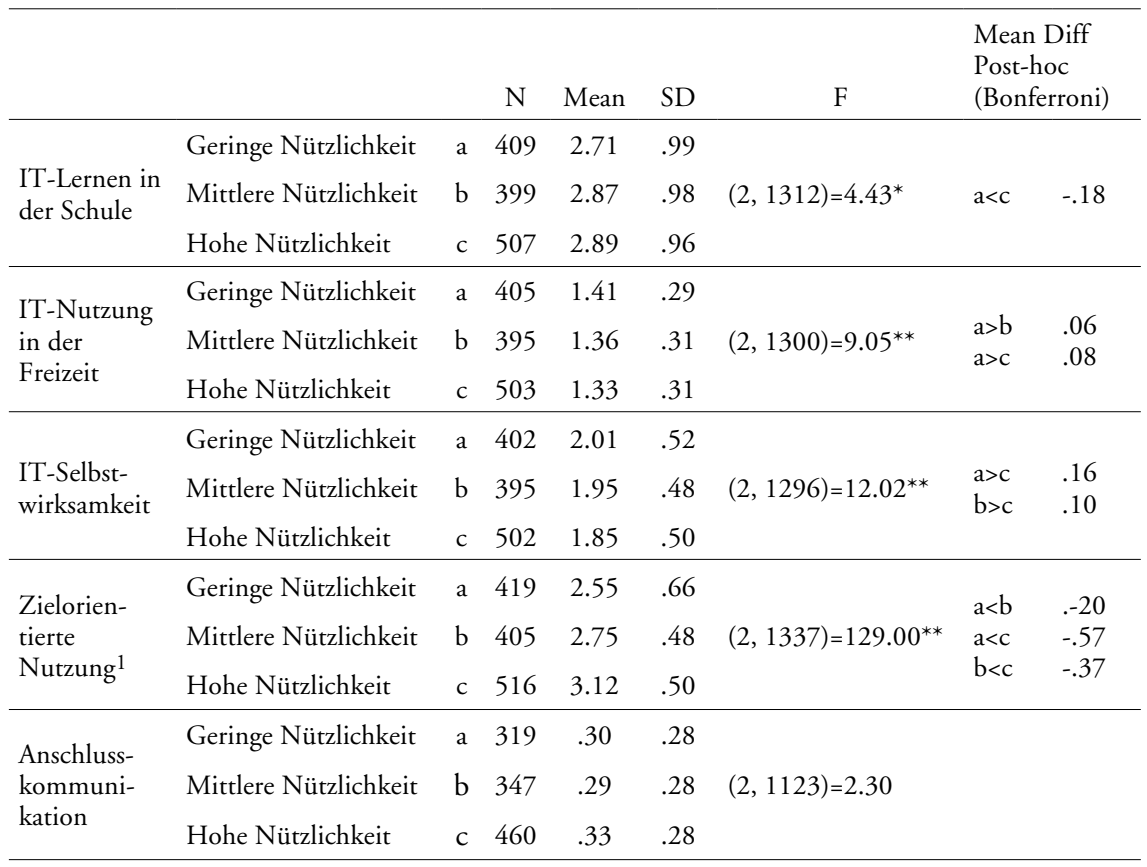

$* \mathrm{p}<.05$

** $\mathrm{p}<.01$

${ }^{1}$ Bei dieser Variable wurde infolge inhomogener Varianzen bei den Post-Hoc-Vergleichen die Games-Howell-Korrektur verwendet.

$\mathrm{Zu}$ fragen bleibt also, ob die IT-Selbstwirksamkeit tatsächlich nur wenig mit der Einschätzung zusammenhängt, nützliche Berufswahlinformationen recherchiert zu haben, oder ob diesbezüglich über andere Ursachen nachzudenken ist, beispielsweise über die Operationalisierung der hier verwendeten Selbstwirksamkeitsskala. Da die Befunde im Kontext der Selbstwirksamkeit auch an anderer Analysestelle wichtig sind, wird auf dieses Merkmal in der Diskussion speziell eingegangen. Hinsichtlich der zielorientierten Nutzung zeigen die Gruppenvergleiche folgendes Muster: Gruppen mit höheren Werten auf der eingeschätzten Nützlichkeit der recherchierten Informationen haben auch höhere Werte auf dem Merkmal des zielorientierten Vorgehens. Insgesamt ist aber darauf hinzuweisen, dass es sich hier um deskriptive Befunde handelt, und dass die Mittelwertdifferenzen relativ gering sind. 


\section{Multivariate Merkmalszusammenhänge}

Um die postulierten, multivariaten Zusammenhänge zwischen den Variablen zu prüfen, wurde ein Strukturgleichungsmodell berechnet. Dieses soll Hinweise darauf liefern, ob die in der Schule aufgebauten bzw. in der Freizeit angeeigneten Recherchekompetenzen eine Auswirkung auf die eingeschätzte Nützlichkeit der recherchierten Berufswahlinformationen haben (vgl. Abb. 2). Wie bereits erläutert, wird angenommen, dass beide Arten von Recherchekompetenzen entweder direkt auf die Nützlichkeit wirken, oder ihre Einflüsse über die Selbstwirksamkeit und die zielorientierte Nutzung vermittelt werden. Zusätzlich wird überprüft, ob eine stattgefundene Anschlusskommunikation die Nützlichkeit der zielorientierten Nutzung mediiert. Das Strukturgleichungsmodell wurde mit Mplus 7.11 berechnet (Muthén \& Muthén, 2017). Da sowohl intervallskalierte als auch dichotome Indikatorvariablen Verwendung finden, wurde ein Weighted-Least-Squares Schätzverfahren (WLSM) mit Delta-Parametrisierung gewählt. Bei diesem Vorgehen werden die fehlenden Werte gemäss einem Maximum-Likelihood-Verfahren geschätzt (Asparouhov \& Muthén, 2010). Gemäss den theoretischen Vorarbeiten wurde das in Abb. 2 dargestellte Modell getestet, wobei vor dem Strukturgleichungsmodell zunächst ein Messmodell mit den in der Berechnung enthaltenen latenten Variablen analysiert wurde. Um den Einfluss von Geschlecht, HISEI und Alter zu kontrollieren wurden sie als unabhängige Variablen für alle endogenen Variablen des Strukturgleichungsmodells verwendet (vgl. Abb. 2).

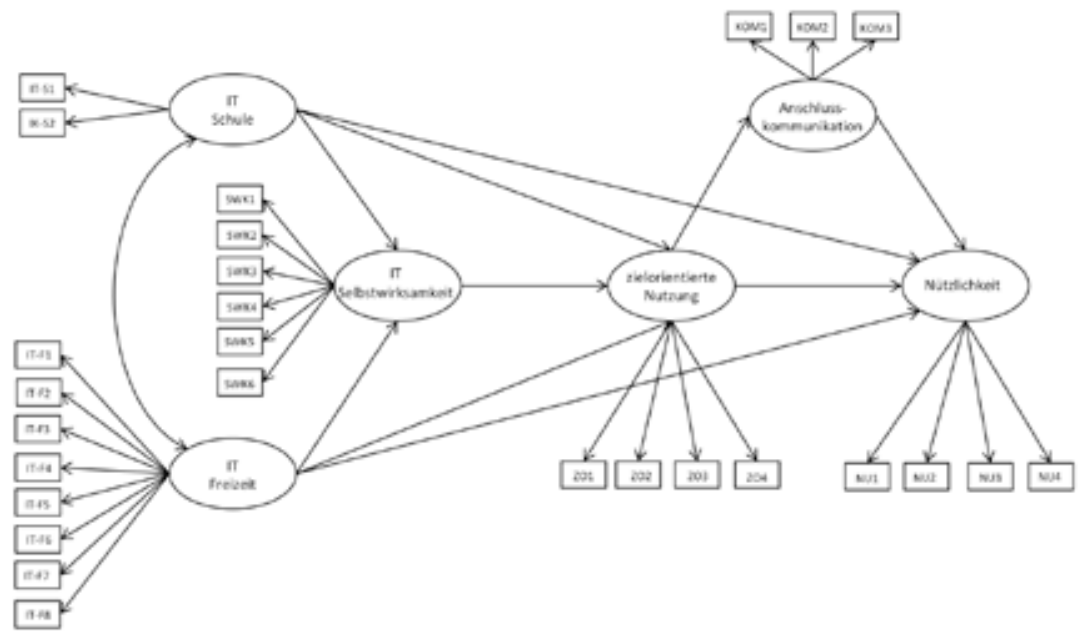

Abb. 2: Theoretisch konzipiertes Strukturgleichungsmodell zur Überprüfung des Nutzens des Internets als Informationsquelle im Berufswahlprozess. Der HISEI, das Geschlecht und das Alter flossen als Kontrollvariablen ins Modell ein. 
Damit geprüft werden kann, ob der Zusammenhang der IT-Recherchekompetenzen auf die Nützlichkeit über eine höhere IT-Selbstwirksamkeit bzw. ein zielorientiertes Vorgehen vermittelt wird, erfolgt zuletzt ein Modellvergleich des oben beschriebenen Modells mit einem Modell, bei dem die direkten Pfade der beiden Formen von IT-Recherchekompetenzen auf die Nützlichkeit entfernt wurden.

\section{Resultate des Strukturgleichungsmodells}

Nach Berechnung des Messmodells wurde eine hohe Kollinearität der Indikatorvariablen der Anschlusskommunikation festgestellt. Deshalb wurde bei der Berechnung des Strukturgleichungsmodells die Anschlusskommunikation mit der Lehrperson als manifeste Variable verwendet. Zusätzlich wurde ein Indikator-Item der IT-Selbstwirksamkeit entfernt, da es im Vergleich mit den anderen Variablen eine niedrige Faktorladung aufwies, und es sich auch inhaltlich von den anderen Indikator-Items abgrenzen liess, da es mathematische Kompetenzen beinhaltete ("Wie gut kannst Du eine Tabellenkalkulation verwenden, um Berechnungen durchzuführen, Daten zu verwalten und Graphen zu zeichnen?»). Nach diesen Anpassungen erreichte das Strukturgleichungsmodell eine gute Anpassungsgüte $(\mathrm{CFI}=.96$; TLI = .95; RMSEA <.03) (vgl. Tab.2).

Allem voran und auch relativ deutlich zeigt sich, dass die direkten Pfade der schulisch bzw. freizeitlich aufgebauten Recherchekompetenzen nicht substantiell mit der eingeschätzten Nützlichkeit der erhaltenen Informationen zusammenhängen. Weder die in der Schule noch die während des freizeitlichen Gebrauchs aufgebauten Recherchekompetenzen sind somit direkt mit der eingeschätzten Nützlichkeit der recherchierten Berufswahlinformationen assoziiert. Obwohl theoretisch davon ausgegangen werden kann, dass diese beiden Dimensionen massgeblich dazu beitragen, dass Lernende nützliche Sachinformationen aus dem Internet gewinnen können, scheinen sie im Zusammenhang mit dem internetbasierten Aufbau von Berufswahlwissen wenig bedeutsam. Beide Quellen der Recherchekompetenzen hängen aber mit der zielorientierten Nutzung statistisch bedeutsam zusammen, wobei der Effekt eher gering ist. Zudem zeigt sich zwischen den schulisch aufgebauten Recherchekompetenzen und der IT-Selbstwirksamkeit ein bedeutsamer, negativer Zusammenhang, während er sich zwischen der freizeitlichen Nutzung des Internets und der IT-Selbstwirksamkeit statistisch nicht absichern liess. Mehr schulisch aufgebaute Recherchekompetenzen hängen also mit einer tieferen IT-Selbstwirksamkeit zusammen, ein Befund, der auf den ersten Blick überrascht, und der, wie bereits weiter oben angesprochen wurde, in der Diskussion spezifisch betrachtet wird. Die engste Verbindung zur eingeschätzten Nützlichkeit des recherchierten Berufswahlwissens weist die zielorientierte Nutzung aus. Je stärker Lernende den Eindruck haben, bei der Informationssuche zielführend vorzugehen, desto eher erscheinen ihnen die erhaltenen Informationen als nützlich. 
Tabelle 2: Ergebnisse des Strukturgleichungsmodells zum Nutzen des Internets im Berufswahlprozess

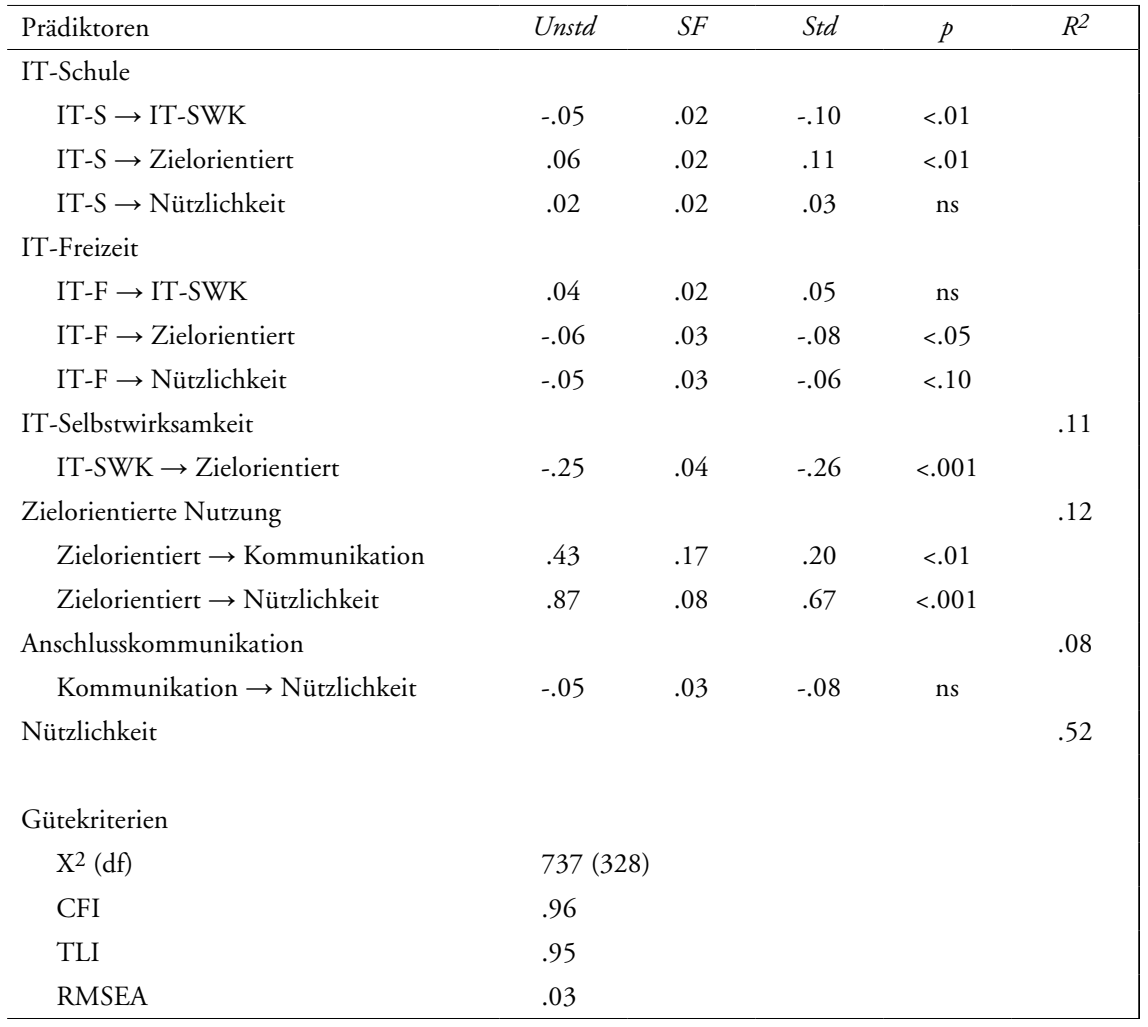

Anmerkungen: $N=1556$; Unstd. $=$ Unstandardisierter Koeffizient; $S F=$ Standardfehler; Std. $=$ Standardisierter Koeffizient; $p=\mathrm{p}$-Wert; $R^{2}=$ Anteil erklärter Varianz; IT-S = IT-Anwendung in der Schule; IT-F = IT-Anwendung in der Freizeit; CFI = Comparative Fit Index; TLI = Tucker-Lewis-Index; RMSEA = Root Mean Square Error of Approximation.

Somit besteht die stärkste Assoziation zwischen zwei Variablen, die konkret an das Berufswahlwissen und damit direkt an den zu recherchierenden Inhalt gebunden sind. Man kann nun darüber nachdenken, inwiefern eher generelle Recherchekompetenzen bzw. eher allgemeine Sucherfahrungen tendenziell wenig dazu beitragen, dass Lernende der Meinung sind, nützliche Informationen zu einem spezifischen Sachbereich wie der Berufswahl erhalten zu haben. Vielleicht hat dies damit zu tun, dass sie zuwenig an einen für die Jugendlichen lebensrelevanten Sachinhalt gebunden sind. Unterstützt wird diese Interpretation allenfalls dadurch, dass die Einschätzung von Jugendlichen, im konkreten Sachbezug zielorientiert vorgehen zu können, für die Nützlichkeitseinschätzung wichtiger ist, als grundlegende Recherchekompetenzen, die sie in der Freizeit oder in der Schule aufgebaut haben. 
Vergleicht man an dieser Stelle nun das eben besprochene Modell mit einem Modell ohne direkte Effekte der IT-Recherchekompetenzen (IT-Schule und IT-Freizeit) auf die eingeschätzte Nützlichkeit, findet sich ein signifikanter Unterschied $\left(\chi^{2}(2, N=1606)=7.39, p<.05\right)$. Das Modell ohne direkte Pfade ist also signifikant schlechter als jenes mit den beiden Pfaden. Wird jedoch nur der direkte Pfad von der IT-Nutzung in der Freizeit auf die Nützlichkeit entfernt, so besitzt dieser Unterschied nur die Tendenz zur Signifikanz $\left(\chi^{2}(1, N=1606)\right.$ $=2.51, .1>\mathrm{p}>.05)$. Dabei ist zu beachten, dass sich die Werte von $\chi^{2}$ mit zunehmender Stichprobengrösse erhöhen, weshalb einige Autoren besonders bei grossen Stichproben vorschlagen, beim Vergleich von komplexeren mit einfacheren Modellen einen kritischen relativen $\chi^{2}$-Wert von 3 zu wählen (Hooper, Coughlan \& Mullen, 2008; Kline, 2005). Unter dieser Annahme ist das Modell mit nur einem direkten Pfad (IT-Freizeit) zu bevorzugen. Insgesamt gilt jedoch, dass Lernende, die davon überzeugt sind, dass ihnen eine auf berufs- bzw. vertiefte Selbstkenntnisse bezogene Informationssuche im Internet zielführend gelingt, tendenziell auch den Eindruck äussern, nützliches Wissen recherchiert zu haben. Dieser Zusammenhang wird nicht durch eine allfällige Besprechung der recherchierten Suchergebnisse mit Eltern, Lehrpersonen oder Peers vermittelt (Anschlusskommunikation).

\section{Diskussion}

Die Befunde lassen sich auf der Folie von theoretisch-konzeptionellen Bezugspunkten, schulpraktischen Relevanzfragen sowie hinsichtlich der Qualität von im Internet angebotenen Informationen besprechen. Dabei steht nicht durchwegs die Berufswahlthematik im Zentrum, sondern wird teilweise über sie hinausgegangen, so dass sich im Zusammenspiel dieser drei Perspektiven auch differenziertere Verständnisse oder weiterführende Fragen ergeben können.

\section{Theoretisch-konzeptionelle Erweiterungen}

Theoretisch-konzeptionell bietet sich das wert-erwartungstheoretische Modell sensu Wigfield und Eccles (2000) an, die Befunde in einem weiteren Rahmen einzuordnen. Anknüpfungspunkt ist die Nutzungskomponente dieses Modells, weil diese mit der hier erfassten Überzeugung von Berufssuchenden in Verbindung gebracht werden kann, im Internet nützliche Informationen recherchiert zu haben. Es ist ein Nutzen, der mit Blick auf aktuelle oder künftige Ziele einer Aufgabe wahrgenommen wird und deshalb nicht nur bezüglich zu treffender Bildungsentscheidungen zentral ist, sondern auch hinsichtlich zielgerichteter Aktivitäten von Lernenden ganz allgemein (Maaz, Hausen, McElvany $\&$ Baumert, 2006). Dies ist bedeutsam, weil es gerade im Berufswahlprozess nicht nur darum geht, dass Heranwachsende einen möglichst passenden Anschlussweg finden, sondern weil sie im Übergangsprozess auch ihre Eigenständigkeit und 
ihre persönliche Handlungsfähigkeit erfahren können. Sich für einen Beruf zu entscheiden ist also, wenn es aus einem lebensbiografischen Horizont betrachtet wird, ein weitgehend neues und auch wichtiges Lernfeld für Schulabgehende, das ihnen ermöglicht, grösser werdende Autonomie zu leben und zunehmende Emanzipation zu erfahren. Dazu können nützliche Informationen einen substantiellen Beitrag liefern, weil sich Lernende, wenn sie überzeugt sind, über hilfreiche Informationen zu verfügen, weitere Aktivitäten und intensives Engagement zutrauen, so dass sie in der Folge produktive Entwicklungsprozesse voranbringen können. Dabei ist aber interessant, dass die Anschlusskommunikation mit den Lehrpersonen, zumindest in der vorliegenden Untersuchung, keinen Zusammenhang mit der eingeschätzten Nützlichkeit der erhaltenen Informationen hat. Dies heisst aber nicht, dass die Lernenden keine Anschlusskommunikation durchführen, sondern es kann bedeuten, dass ein allfälliger Austausch mit den im Internet recherchierten Informationen vielleicht nicht primär mit Lehrpersonen stattfindet. Möglicherweise tauschen sich Heranwachsende auch mit anderen Personen darüber aus, ob die im Internet gefundenen Informationen für ihren Berufswahlprozess gehaltvoll sind, gegebenenfalls mit ihren Kolleginnen oder Kollegen oder mit ihren Eltern. Vielleicht zeigt sich in diesem Befund aber auch so etwas wie ein naives Nutzungskonzept, oder, gerade anders herum, eine hohe, vielleicht auch eine übersteigerte Nutzungssicherheit. Mit einem naiven Nutzungskonzept könnte einhergehen, dass Lernende recherchiertes Wissen grundsätzlich wenig hinterfragen, es also weitgehend kritiklos als gültig und verlässlich annehmen. Daher gibt es wenig Notwendigkeit, erhaltene Informationen mit jemandem diskursiv zu prüfen. Eine hohe Nutzungssicherheit hingegen könnte heissen, dass die Lernenden weitgehend davon überzeugt sind, über ausreichende Fähigkeiten zu verfügen, zielführend, eigenständig und gleichzeitig informationskritisch durchs Netz navigieren zu können und deshalb einen Anschlussdiskurs mit ihren Lehrpersonen für nicht notwendig erachten. Neben diesem Anschlusskommunikations-Effekt ist der tendenzielle Zusammenhang zwischen freizeitlich aufgebauten Recherchekompetenzen und der eingeschätzten Nützlichkeit der erhaltenen Informationen nicht minder bedeutsam, besonders deshalb nicht, weil er zwischen den schulisch aufgebauten Recherchekompetenzen und der eingeschätzten Nützlichkeit auch der Tendenz nach nicht gefunden werden konnte. Ob eine im freizeitlichen Gebrauch vielleicht höhere inhaltliche Relevanz diesen Unterschied erklären kann, lässt sich an dieser Stelle nicht abschliessend klären. Wichtig aber ist festzuhalten, dass Informationen vor allem dann als nützlich betrachtet werden, wenn die Lernenden davon überzeugt sind, bei den Recherchen zielorientiert vorgehen zu können. Dies könnte mit konkretem Blick auf den schulischen Unterricht von Bedeutung sein, insbesondere, wenn im Kontext der Zielorientierung auch die IT-Selbstwirksamkeit betrachtet wird. 
IT-Unterricht und Internetnutzung zum Wissensaufbau

Im Rahmen des nun etwas stärker schulisch orientierten Diskussionsaspekts gilt es, die Befunde zur IT-Selbstwirksamkeit im Speziellen zu betrachten. Einerseits ist unerwartet, dass der Zusammenhang zwischen schulisch aufgebauten Recherchekompetenzen und der erfassten IT-Selbstwirksamkeit negativ ist. Dies scheint auf den ersten Blick erstaunlich, weil in Anlehnung an das weiter oben Gesagte eher anzunehmen wäre, dass höhere Recherchekompetenzen mit einer höheren IT-Selbstwirksamkeitserwartung verknüpft sind. Vielleicht lässt sich die leicht negative Verbindung aber dadurch erklären, dass, wie dargestellt wurde, die Recherchekompetenzen nicht in einem berufswahlspezifischen Anwendungsbezug erworben und erhoben wurden, sondern in aufgabenbasierten Lernsettings ohne berufswahlspezifischen Inhaltsbezug. Eventuell spielt hier aber auch hinein, dass nicht ganz unbestritten ist, ob das international als IT-Selbstwirksamkeits-Skala bezeichnete und eingesetzte Instrument, tatsächlich IT-Selbstwirksamkeit erfasst, oder ob es nicht eher aufgabenorientierte Kompetenzen abfragt. Ungeachtet dieser offenen und weiter zu klärenden Fragen ist zudem daran zu denken, dass die Art der Informationssuche im Kontext der Berufswahl anders geartet sein könnte, als in anderen Sachbezügen. Während in letzteren kumulativer Wissensaufbau vielleicht direkter möglich ist, spielt im Prozess der Berufswahl stets mit, dass sich die Suchbedingungen durch gefundene Informationen laufend ändern können. Immer wieder neu aufgebautes oder sich weiter transformierendes Wissen kann inhaltliche Einsichten in Bereiche öffnen, die den Lernenden vielleicht bislang nicht oder nur wenig im thematischen Bewusstsein waren.

Und über dieses Phänomen der Berufswahldynamik hinausgehend ist auf Grund der Befunde für den schulischen Kontext vielleicht noch zu diskutieren, dass im Rahmen des internetbasierten Aufbaus von Berufswahlwissen, die in der Schule erworbenen Recherchekompetenzen weitgehend beziehungslos zur eingeschätzten Nützlichkeit des recherchierten Wissens stehen. Zwar unterstützen sie die Meinung der Lernenden, zielorientiert vorgehen zu können, was heisst, dass sie, je häufiger sie in der Schule Recherchekompetenzen aufgebaut haben, eher sagen, im Internet Berufswahlwissen zielorientiert suchen zu können. Für den Entscheid aber, ob die recherchierten Informationen auch nützlich sind, erweisen sie sich jedoch als weitgehend bedeutungslos. Es scheint also, und das kann für den schulischen IT-Unterricht wichtig sein, dass beim Aufbau der Fähigkeit, nützliche Informationen im Internet recherchieren zu können, vor allem der zielorientierte Inhaltsbezug wichtig ist. Die eingangs erwähnte Beziehung zwischen allgemeinem Anwendungswissen als Voraussetzung dafür, spezifisches Sachwissen zielführend und nutzenorientiert aufzubauen, lässt sich vielleicht theoretisch nachvollziehen, konnte hier aber nicht belegt werden. Die Verbindung zur eingeschätzten Nützlichkeit scheint jedoch enger zu werden, wenn Lernende näher am Inhalt sind, wenn sie also beispielsweise der Überzeugung sind, mit Blick auf einen spezifischen und für sie inhaltlich 
bedeutsamen Wissensaufbau (beispielsweise Berufswahlwissen) das Internet zielführend nutzen zu können. So liesse sich darüber nachdenken, inwiefern der IT-Unterricht dies aufnehmen könnte, beispielsweise indem Wissensrecherchen vor allem im Rahmen von realen und relevanten Nutzungsanforderungen erfolgen. Konkret heisst dies, dass Recherchekompetenzen zur Unterstützung von Wissensaufbau in einem spezifischen Feld nicht nur als funktionale Aspekte thematisch übergreifend gelernt werden sollten, sondern vorteilsweise auch auf der Folie eines realen und möglichst relevanten Handlungskontexts voranzubringen sind.

\section{Qualität der zur Verfügung stehenden Informationen} Abschliessend lässt sich mit Blick auf die Qualität der angebotenen Informationen ein weiteres und für den schulischen Unterricht ebenfalls relevantes Diskussionsfeld ansprechen. Dass den Lernenden umfangreiche Angebote zur Verfügung stehen, sich berufswahlspezifisches Wissen über die eigene Person oder die Berufswelt anzueignen, steht ausser Frage. Lehrstellennachweise seitens kantonaler Berufsbildungsämter oder vom Bund unterhaltene Web-Seiten zu branchenspezifischen Anforderungsprofilen bzw. zu Angaben, die über die Bedeutung einzelner Schulfächer für das Erlernen eines spezifischen Berufs Auskunft geben, bzw. Möglichkeiten, individuelle Kompetenzbilanzierungen am Internet vorzunehmen, sind nur einige wenige Beispiele, die zeigen, dass im Berufswahlbereich Wissen im Netz umfassend zur Verfügung steht und verbreitet wird. Die eingeschätzte Nützlichkeit hängt nun aber nicht nur von der Fähigkeit ab, mit dem Internet als Informationsmedium zielführend und nutzenbringend umgehen zu können, sondern es spielt sehr wahrscheinlich auch die Qualität der dargebotenen Informationen eine Rolle, also die so genannte Anwenderfreundlichkeit von entsprechenden Web-Seiten. Allenfalls liesse sich, auf Grund der vorliegenden Befunde darüber nachdenken, inwiefern Informationen so aufbereitet werden könnten, dass sie vor allem jenen zugutekommen, die wenig zielführend vorgehen können, so dass auch sie inhaltlich gehaltvolle Wege finden, um zu unterstützenden Informationen zu gelangen, die ihnen die lebensbiografisch zentrale Einmündung in die berufliche Ausbildung erleichtern können.

Zugegebenermassen nehmen diese drei Diskussionspunkte drei ausgewählte Aspekte in den Blick, die allenfalls als Denkrichtung weitere Fragen generieren oder vielleicht auch unterrichtliche Prozesse in gewissen Punkten weiterentwickeln helfen. Es ist aber klar, dass hier thematisch nur ein kleiner Ausschnitt fokussiert werden konnte. So fehlen beispielsweise Hinweise zur Verarbeitungsqualität von recherchierten Informationen genauso, wie es nicht möglich war, ein objektives Mass für die Nützlichkeit der recherchierten Informationen zu definieren. Trotz dieser Limitierungen, zu denen auch eine nicht kausale Lesart des analysierten Modells gehört, gibt die Untersuchung dennoch erste Hinweise zum Zusammenhang von IT-Recherchekompetenzen und der eingeschätzten Nützlichkeit von recherchiertem Sachwissen. Vielleicht kann nun auf dieser 
Basis weiterführende Forschung angeregt oder konkretes Handeln angeleitet werden und somit aktuelles Wissen im Kontext von Internetrecherchen von Jugendlichen erweitert werden.

\section{Literatur}

Asparouhov, T. \& Muthén, B. (2010). Weighted least squares estimation with missing data. LA, CA: Muthén \& Muthén. Retrieved from www.statmodel.com

Beinke, L. (2008). Das Internet- ein Instrument zur Berufsorientierung? Frankfurt a.M.: Peter Lang.

Brown, D., Brooks, L. \& Klostermann, M. (1994). Karriere-Entwicklung. Stuttgart: KlettCotta.

D-EDK. (2016). Lehrplan 21. Luzern: D-EDK.

Düggeli, A. \& Kinder, K. (2013). Ich trau mich schon und kann das auch: Jugendliche bewältigen Berufswahlbesorgnisse mit ihren Lehrpersonen. In T. Brüggemann \& S. Rahn (Hrsg.), Berufsorientierung (S. 211-219). Münster: Waxmann.

Düggeli, A., Kinder, K. \& Bergamin, P. (2013). Informations- und Computerkompetenz im Kontext der beruflichen Orientierung von Schülerinnen und Schülern der Sekundarstufe I. In A. d. W. Schweiz (Hrsg.), Förderung der MINT-Kompetenzen zur Stärkung des Bildungs-, Wirtschafts- und Wissenschaftsstandorts Schweiz (S. 107-113). Bern: Akademien der Wissenschaften Schweiz.

Fraillon, J., Ainley, J., Schulz, W., Friedman, T. \& Gebhardt, E. (2014). Preparing for life in a digital age: The IEA International Computer and Information Literacy Study international report. Cham: Springer.

Fraillon, J., Schulz, W., Friedman, T., Ainley, J. \& Gebhardt, E. (2015). International Computer and Information Literacy Study: ICILS 2013: Technical report. Cham: Springer.

Ganzeboom, H. B. G., De Graaf, P. M., Treiman, D. J. \& De Leeuw, J. (1992). A standard international socio-economic index of occupational status. Social Science Research, 21, (1), $1-56$.

Golisch, B. (2002). Wirkfaktoren der Berufswahl Jugendlicher: eine Literaturstudie. Frankfurt am Main: Peter Lang GmbH.

Hellberg, B. M. (2009). Entscheidungsfindung bei der Berufswabl: Prozessmodell der Emotionen und Kognitionen. Wiesbaden: VS Verlag für Sozialwissenschaften.

Herzog, W., Neuenschwander, M. P., \& Wannack, E. (2006). Berufswahlprozess. Bern: Haupt Verlag.

Hirschi, A. (2011). Berufswahlfreiheit als Selbstgestaltung beruflicher Entwicklung. In R. Marty, A. Hirschi, D. Jungo, M. Jungo, \& R. Zihlmann (Hrsg.), Berufswahlfreiheit. Ein Modell im Spannungsfeld zwischen Individuum und Umwelt (S. 99-100). Bern: SDBB Verlag.

Holland, J. L. (1997). Making vocational choices: A theory of vocational personalities and work environments. Odessa, FL: Psychological Assessment Resources.

Hooper, D., Coughlan, J. \& Mullen, M. (2008). Structural equation modeling: Guidelines for determining model fit. Electronic Journal of Business Research Methods, 6, (1), 53-60.

Kline, R. B. (2005). Principles and practices of structural equation modeling. New York, NY: Guilford Press.

Konsortium PISA.ch (2014). PISA 2012: Vertiefende Analysen. Bern und Neuchâtel: SBFI/ EDK und Konsortium PISA.ch.

Lent, R. W., Brown, D. \& Hackett, G. (2002). Social cognitive career theory. In D. Brown (Ed.), Career choice and development (pp. 255-311). San Francisco, CA: Wiley.

Maaz, K., Hausen, C., McElvany, N. \& Baumert, J. (2006). Stichwort: Übergänge im Bildungssystem. Theoretische Konzepte und ihre Anwendung in der empirischen 
Forschung beim Übergang in die Sekundarstufe. Zeitschrift für Erziehungswissenschaft, (3), 299-327.

Mitchell, L. K. \& Krumboltz, J. D. (1994). Die berufliche Entscheidungsfindung als sozialer Lernprozess: Krumboltz`Theorie. In D. Brown \& L. Duane (Hrsg.), Karriere-Entwicklung (S. 157-210). Stuttgart: Klett-Cotta.

Muthén, L. K. \& Muthén, B. (2017). Mplus version 8 user's guide. Los Angeles, CA: Muthén \& Muthén.

Ratschinski, G. (2008). Berufswahlkompetenz. In M. Koch \& P. Straßer (Hrsg.), In der Tat kompetent: Zum Verständnis von Kompetenz und Tätigkeit in der beruflichen Benachteiligtenförderung (S. 73-90). Bielefeld: W. Bertelsmann Verlag.

SKBF. (2018). Bildungsbericht Schweiz 2018. Aarau: Schweizerische Koordinationsstelle für Bildungsforschung.

Wigfield, A. \& Eccles, J. S. (2000). Expectancy-value theory of achievement motivation. Contemporary Educational Psychology, (25), 68-81.

Willemse, I., Waller, G., Süss, D., Genner, S. \& Huber, A.-L. (2012). JAMES-Jugend, Aktivitäten, Medien - Erhebung Schweiz. Zürich: Zürcher Hochschule für Angewandte Wissenschaften.

Schlagworte: Übergang in die Berufsbildung, Berufswahlwissen, IT-Kompetenz

\section{Internet comme source d'information pour augmenter les connaissances nécessaires pour le choix professionnel: comment les jeunes l'utilisent et comment ils évaluent les informations obtenues}

\section{Résumé}

La présente recherche thématise la relation entre l'utilisation ciblée d'internet pour la construction du savoir utile au choix professionnel et l'utilité perçue des résultats de la recherche. Il ressort des données relatives aux apprenants de la huitième classe $(\mathrm{N}=1606)$, qui ont été récoltées en Suisse en 2013 dans le cadre de l'étude IEA ICIL (International Computer and Information Literacy) qu'une recherche ciblée va de pair avec la perception d'avoir développé quelque chose d'utile. Les compétences transmises par l'enseignement scolaire de même que le savoir acquis relatif à l'utilisation d'internet comme source d'informations ainsi qu'un éventuel entretien avec parents ou enseignant(e)s portant sur le nouveau savoir relatif au choix professionnel se sont révélés peu significatifs à l'égard de l'utilité perçue. Les résultats de l'enquête seront discutés du point de vue théorique et conceptionnel concernant leur pertinence pour le choix professionnel ou pour les cours d'informatique.

Mots-clés: Passage à la formation professionnelle, connaissances utiles au choix professionnel, compétences ICT 


\section{Internet come fonte di informazione per acquisire conoscenze di scelta profes-sionale: come usano i giovani Internet e quanto utile valutano le informazioni che ricevono}

\section{Riassunto}

La presente ricerca tematizza la relazione entro l'utilizzo funzionale del internet per la costruzione del sapere utile al riguardo della scelta professionale e dell'utilità percepita dei risultati di ricerca online. Sulla base di dati di alluni dell'ottava classe $(\mathrm{N}=1606)$, che sono stati raccolti in svizzera nel 2013 nel ramo della ricerca IEA ICIL (International Computer and Information Literacy), viene dimostrato, che una ricerca online con una chiara visione degli obbiettivi incide con la percezione di avere sviluppato conoscenze utili. Le competenze trasmesse nel insegnamento scolare come pure il sapere acquisito nel tempo libero al riguardo del utilizzo del internet come fonte d'informazione e anche un eventuale discorso con genitori o insegnanti al riguardo delle nuove conoscenze relative alla scelta professionale si rivelano poco significativi per quanto concerne l'utilità percepita. L'autoefficacia attribuita all'informatica è tuttavia in media correlazione con l'utilizzo del internet nel tempo libero per ottenere delle informazioni e tendenzialmente associata all'utilizzo del internet con una chiara visione degli obbiettivi. Per concludere, i risultati della ricerca sono discussi dal punto di vista teoretico e concezionale riguarda la loro rilevanza per la scelta professionale e l'informatica come insegnamento.

Parole chiave: Transizione alla formazione professionale, conoscenze della scelta professionale, competenze IT

\section{The Internet as a source of information for making a career choice: how young people use it, and how useful they are at evaluating the information they receive}

\section{Abstract}

This study is concerned with the relation between the way learners use online searches on the internet to gain new knowledge about choosing a profession, and the degree to which learners estimate these searches to be useful for them. Data were gathered in Switzerland in 2013 the context of the International Computer and Information Literacy study (IEA ICIL). The sample of $\mathrm{N}=1606$ learners showed that a goal-oriented search for information was significantly related to learners' sense of usefulness while building up new knowledge. By contrast, the following factors proved to be less important concerning learners' own sense of usefulness: skills for using the internet taught at school, skills for using the internet acquired in learners' leisure time, and conversations with parents or 
teachers. In this study, we discuss these findings in the context of value expectancy theory and discuss their relevance concerning learners' choice of future profession, as well as the subject of computer-studies in school.

Keywords: Transition to VET-Education, knowledge for career choice, IT-Competence 
